\title{
CAPITULO OT
}

\section{ESTÁGIO MULTIPROFISSIONAL NO CONTEXTO DO NASF-AB: RELATO DE EXPERIÊNCIA}

\author{
$\underline{\text { Luciana Kelly da Silva Fonseca }}{ }^{1}$, Marina Rufino Mariano ${ }^{2}$, Gisele Cristine Araújo \\ Nascimento $^{3 \text {, Daline da Silva Azevedo }}{ }^{4}$, Kauan Gustavo de Carvalho ${ }^{5}$, Mateus Egilson \\ da Silva Alves ${ }^{6}$, Iana Clara Rodrigues Marques ${ }^{7}$, Maria Gabriela do Nascimento \\ Araújo 8 \\ ${ }^{1}$ Universidade Federal do Piauí/UFPI, (1.kelly_fonseca@hotmail.com) \\ ${ }^{2}$ Universidade Federal do Piaú́/UFPI, (mari.rufi.mr@gmail.com) \\ ${ }^{3}$ Universidade Federal do Piauí/UFPI, (giselecristine04@ outlook.com) \\ ${ }^{4}$ Universidade Federal do Piauí/UFPI, (dalinesilvaazevedo@gmail.com) \\ ${ }^{5}$ Universidade Federal do Piauí/UFPI, (kauancarvalho2008@gmail.com) \\ ${ }^{6}$ Universidade Federal do Delta do Parnaíba/UFDpar, (mateusegalves@gmail.com) \\ ${ }^{7}$ Universidade Federal do Piauí/UFPI, (ianaclara51@ @otmail.com) \\ ${ }^{8}$ Universidade Federal do Delta do Parnaíba/UFDpar, (gabi.sikver@gmail.com)
}

\section{RESUMO}

Objetivo: Este relato de experiência tem o objetivo de aludir sobre as observações de estudantes da área da saúde acerca da experiência de estágio observacional em uma equipe do Núcleo Ampliado de Saúde da Família e Atenção Básica (NASF-AB) em um município do estado do Ceará, nos anos de 2016-2018. Método: Esta produção trata-se de um relato de experiência das atividades realizadas durante um estágio observacional sem remuneração e não-obrigatório. As atividades desenvolvidas foram prosseguidas de anotações e registros em cadernos com descrição das ações realizadas no dia-a-dia, bem como atividades de observação. O estágio observacional foi realizado com equipe multiprofissional, composta por psicólogo, fisioterapeuta, nutricionista e profissional de educação física, nos intervalos entre os semestres acadêmicos, assim, atividades abrangeram diversas temáticas no âmbito da atenção básica e trabalho intersetorial. Resultados: A vivência da realidade do SUS, mais especificamente da

\section{E - book Pesquisa e Tecnologia: Protagonismo e Inovações}


Atenção Básica em saúde, ainda na graduação é de suma importância para uma formação mais humana e resolutiva enquanto acadêmicos de saúde, direcionando o olhar para a clínica ampliada. Enquanto olhar acadêmico de aprendiz, a cada dia era uma oportunidade de vivenciar algo novo, isto é, em todas as reuniões, visitas domiciliares e oficinas de educação em saúde, sempre houve algo a assimilar. Tais questões assumiram um lugar de reiteração e incentivo para a aquisição de conhecimentos. Conclusões: É preciso refletir e agir em favor de uma concepção de clínica ampliada. Ademais, é oportuno realizar projetos, desenvolvendo uma prática única e ao mesmo tempo plural, em proveito dos indivíduos e comunidades assistidas e referenciadas.

Palavras-chave: Atenção básica; NASF-AB; Estágio observacional.

Área Temática: Saúde Pública.

E-mail do autor principal: 1.kelly_fonseca@ hotmail.com

\section{INTRODUÇÃO}

A criação do Sistema Único de Saúde (SUS) em 1988, fez emergir diversas ações no âmbito da saúde que encontram-se em curso de renovação, amparado, pelas diretrizes e princípios que orientam a composição e reconhecimento da saúde como bem social. Destarte, dentro das reformulações no modelo assistencial estabelecidas ao longo dos tempos no SUS, encontra-se a Política Nacional de Atenção Básica (PNAB) que vem sendo estabelecida visando proporcionar e garantir acesso coletivo à saúde. Assim, diversos projetos foram implementados no país, com ênfase atrelada à Atenção Básica de Saúde, como a Estratégia Saúde da Família (ESF) e o Núcleo de Apoio a Saúde da Família e Atenção Básica (NASF-AB) (FERRI; PEREIRA; MISHIMA; CACCIA-BAVA e ALMEIDA, 2007; FERREIRA e LADEIA, 2018).

A Atenção Básica em Saúde (ABS) possui, entre suas principais características, a produção de cuidado em saúde de forma descentralizada, regionalizada e territorial seja para o indivíduo, sua família e coletividade. Portanto, a estratégia da ABS propõe uma ordenação e coordenação nos cuidados em todos os níveis de atenção (BRASIL, 2017; GIRÃO; GOMES; MAIA, 2020; GIOVANELLA, 2018; RODRIGUES et al., 2014). As ações implementadas dentro da ABS estão diretamente implicadas no fazer das equipes de saúde multiprofissionais, como as que constituem serviços como Unidades Básicas de Saúde (UBS), Centros de Saúde da Família (CSF), dentre outros, cuja atuação tem se norteado por meio de territórios e clientela adscrita.

\section{E - book Pesquisa e Tecnologia: Protagonismo e Inovações}


Destarte, O NASF-AB foi criado pela Portaria n ${ }^{\circ}$ 154/GM de 24 de janeiro de 2008 visando potencializar a capacidade das ESF, de forma a responder às deficiências dos usuários abrangidos pelo território definido para cada equipe. Esse equipamento, encontra-se baseado nos princípios da integralidade e da interdisciplinaridade, dispondo de uma equipe multidisciplinar que tem como finalidade o auxílio no atendimento multidisciplinar das demandas dos usuários dos serviços da ESF (SOUZA, et al., 2013).

Contudo, a PNAB vigente fomentou a revisão de diretrizes da equipe de Atenção Básica e diante de demandas emergentes algumas mudanças ocorreram em tal equipamento, a exemplo, o nome do dispositivo deixou de ser Núcleo de Apoio à Saúde da Família (NASF) e passou a ser Núcleo Ampliado de Saúde da Família e Atenção básica (NASF-AB), para que pudesse aumentar e equiparar as ações da Atenção Básica e ampliando a capacidade de resolutividade de questões dos usuários do SUS, entre outros. Sendo assim, passar a ser parte integrante da equipe de unidade de saúde fornecendo um serviço ampliado (CURY, RODACOSKI, SANTOS, 2019). Logo, o serviço foi modificado no sentido de lidar com alguns desafios que o SUS enfrenta, como a resolubilidade e a integralidade.

Deste modo, os diferentes profissionais devem estabelecer e compartilhar saberes, práticas e gestão do cuidado, com uma visão comum, e aprender a solucionar problemas pela comunicação, de modo a maximizar as habilidades singulares de cada um (PNAB, 2017). Assim, profissionais que fazem parte do NASF-AB são psicólogo, fisioterapeuta, nutricionista, profissional de educação física e etc. Tais profissionais desenvolvem reuniões, orientações em saúde, matriciamento das equipes, atendimentos individuais, atendimentos e visitas domiciliares, grupos e atividades coletivas, entre outros (OLIVEIRA, ROCHA, CUTOLO, 2012).

Essa nova área estimula esses profissionais atuarem numa lógica de matriciamento, operando um trabalho diferenciado que deve ser conhecido desde a graduação. Sendo válido enfatizar que a ocupação na saúde caracteriza-se a partir da utilização de ciências leves, que são aquelas vinculadas ao campo relacional e que se desenham em diversos modos de se comunicar com o usuário, na produção de vínculo, na realização de escuta qualificada e acolhimento. Orientando-se pela dialética de corresponsabilização e direção inteirada do cuidado, através de projetos terapêuticos e assistência aos usuários, considerando a singularidade dos sujeitos (CAMPOS, 2005; GONÇALVES, LANCMAN, SZNELWAR, et. al., 2015).

Destarte, considerando tal proposta, este relato de experiência tem o objetivo de aludir sobre as observações de estudantes da área da saúde acerca da experiência de estágio observacional em uma equipe do Núcleo Ampliado de Saúde da Família e Atenção Básica (NASF-AB) em um município no estado do Ceará, nos anos de 2016-2018. Com o intuito de

\section{E - book Pesquisa e Tecnologia: Protagonismo e Inovações}


direcionar e trazer considerações acerca do estágio observacional no campo da saúde, visando instruir futuros profissionais e acadêmicos focando em aspectos elementares do equipamento, como o matriciamento, atendimentos individuais, trabalhos com grupos e etc.

\section{MÉTODO}

Esta produção trata-se de um estudo descritivo, no formato de relato de experiência, das atividades realizadas durante um estágio observacional não obrigatório sem remuneração e de demanda espontânea, em uma cidade do Ceará, nos anos de 2016-2018. O estágio supervisionado observacional foi realizado com a equipe multiprofissional do antigo NASF, hoje NASF-AB-1, composta por psicólogo, fisioterapeuta, nutricionista, assistente social e profissional de educação física. As atividades abrangeram diversas temáticas no âmbito da atenção básica e trabalho intersetorial. O município possui cerca de 52 mil habitantes, com aproximadamente 16 Unidades Básicas de Saúde (UBS's), no qual a equipe do NASF era responsável por 10 equipes.

O estágio teve duração de três meses entre os anos de 2016-2018 sendo vivenciado entre os intervalos dos semestres da graduação dos estagiários em questão alternadamente. As atividades desenvolvidas foram prosseguidas de anotações e registros em cadernos com descrição das ações realizadas no dia-a-dia. Assim, as atividades de observação se deram de forma integral, ou seja, de forma multidisciplinar atuando na lógica da atenção integral do usuário do SUS, configurando-se assim como uma experiência pluralizada e rica em detalhes de como a saúde se figura e está inscrita na atual Atenção Básica de Saúde. Destarte, foram cumpridas pela equipe do NASF as subsequentes condutas: atividades em grupos, oficinas de orientações em saúde, visitas domiciliares, atendimento domiciliar compartilhado, educação permanente em saúde e discussão do Projeto Terapêutico Singular (PTS).

A técnica utilizada neste trabalho teve como referência principal VIANNA (2003) que disserta sobre o estágio observacional em seus escritos. Então, segundo tal autor este relato figura-se como observação aberta, onde estagiário e preceptores estavam sabendo da presença de ambos no local; não participante, ou seja, não ouve envolvimento nas atividades exercidas pelos profissionais; observação não estruturada, em que não seguia-se roteiro e os fatos foram registrados sem análise de repertório comportamental. Sendo importante ressaltar que o estágio observacional é uma brecha para reflexão, conhecimento e introdução dos profissionais na área em que esboça interesse.

\section{E - book Pesquisa e Tecnologia: Protagonismo e Inovações}




\section{RESULTADOS E DISCUSSÃO}

A vivência da realidade do SUS, mais especificamente da atenção básica em saúde, ainda na graduação é de suma importância para uma formação mais humana e resolutiva enquanto acadêmicos de saúde em geral, direcionando o olhar para a clínica ampliada. Enquanto olhar acadêmico de aprendiz, a cada dia era uma oportunidade de vivenciar algo novo, isto é, em todas as reuniões, visitas domiciliares e oficinas de educação em saúde, sempre houve algo a assimilar. Tais questões assumiram um lugar de reiteração e incentivo para a aquisição de conhecimentos. Com essa experiência, pode-se dizer que para poder realizar um trabalho eficiente na Atenção Básica, o primeiro passo é ter clareza a respeito do tipo de serviço a ser desenvolvido, não deve ser apenas clínico, ou seja, aquele tradicional.

Assim, o modo de trabalho na Atenção Básica (AB) é assinalado por uma submersão no território, atuação centrada no contexto das famílias, longitudinalidade, ordenação e coordenação do cuidado, estabelecimento de vínculo, tornando a $\mathrm{AB}$ um campo de atuação privilegiado para os profissionais em saúde das mais variadas especialidades (MOTTA; CARVALHO, 2015). Sendo assim, os estagiários tiveram como foco a observação da imersão desses atores da saúde em seu contexto de trabalho com o intuito de conhecer a rede de saúde e ter familiaridade com os serviços ofertados pela equipe alvo da observação deste estágio.

Os profissionais do NASF AB-1 cobriam cerca de 10 equipes de ESF sendo distribuídos em todo o território da cidade, seja ele urbano e/ou rural. Distribuíam seu processo de trabalho durante a semana em variados arranjos organizacionais de trabalho para que nenhuma equipe que solicitasse seu apoio ficasse sem auxílio na questão solicitada. Nesse sentido, as funções eram desempenhadas tanto em ordem de atendimento individual, como grupal com os usuários, bem como com as equipes de ESF. Destarte, as ações que foram desenvolvidas no tempo em que o estágio observacional aconteceu foram: oficinas de orientação em saúde, visitas domiciliares, atendimentos domiciliares compartilhados, estruturação compartilhada de diagnósticos e terapêuticas.

Assim, as oficinas de orientações em saúde aconteciam sempre que os profissionais do NASF AB-1 eram requisitados, ou seja, sempre que uma equipe de Saúde da Família sentia necessidade de auxilio em determinado dia com foco em alguma temática, os profissionais eram acionados no sentido de auxiliar com seus conhecimentos a respeito de uma temática em especifico. Tal ação procura deslocar-se no sentido de conceder ao usuário estabelecer vinculo e uma relação de cuidado consigo mesmo, de afetividade para com os outros, o que vem de encontro ao perfil político-social da clínica ampliada (MENDONÇA, 2005). Sendo importante

\section{E - book Pesquisa e Tecnologia: Protagonismo e Inovações}


salientar que foi constatado pelos estagiários que as oficinas em questão tinham bastante adesão da população, com participação ativa e vivaz, tendo como principal caraterística formular e implementar um pensamento crítico entre os participantes das oficinas para o reconhecimento de seus problemas e na atuação de forma individual e coletiva para solucionar possíveis problemas, sendo possível observar a autonomia dos usuários do SUS sendo valorizada como de fato deve ser.

No que diz respeito as visitas domiciliares, estas aconteciam de maneira programada e acordada entre a gestão das unidades básicas, que eram as responsáveis por fornecer a forma de locomoção dos profissionais, bem como com os familiares que iriam receber tal ação em saúde em suas casas. Esta modalidade de atendimento se configura em uma atuação voltada para a saúde individual de pessoas que não podiam se deslocar de alguma maneira para a sua unidade de referência. Neste ponto do processe de trabalho pode-se perceber um manejo de saúde coletiva, pois na ocasião as demandas dos familiares eram atendidas de maneira intencional ou não. No locomover-se até as casas dos usuários era importante no sentido de reconhecimento de território e demandas que existiam extra muros das unidades básicas de saúde, figurando-se em uma relevante ação que o SUS pressupõe que é o fazer saúde em território, entrelaçando-se com maior equidade da assistência em saúde, além de fortalecimento de vínculo com a equipe que leva saúde até esses indivíduos.

Algumas dificuldades foram encontradas nesse tipo de intervenção, como o processo de acesso ao local da visita pelo profissional, muitas vezes faltando transporte. A dificuldade de alguns profissionais encararem como algo benéfico para o vínculos entre paciente e profissional e não apenas como algo que seja de cunho irrelevante. Como cita os autores Paim e Almeida Filho (1998):

A maior aproximação do contexto de vida dos usuários possibilita a valorização da dimensão subjetiva das práticas em saúde, das vivências dos usuários e dos trabalhadores da saúde, abrindo espaços de comunicação e diálogo entre saberes e práticas, além de novas perspectivas para a reflexão e ação.

As visitas domiciliares eram relativamente regulares na sede e nos distritos que ficavam ladeados a sede urbana. Cada dia da semana era planejado para que houvesse visitas nesses distritos afim de não deixar de assistir nenhuma equipe de ESF, todos os profissionais se locomoviam com transporte cedido pela prefeitura da cidade. Os pacientes eram em sua maioria idosos, pessoas que sofrera algum tipo acidente ou crianças com algum transtorno. É importante

\section{E - book Pesquisa e Tecnologia: Protagonismo e Inovações}


destacar, que tal técnica se mostrava eficaz no quesito vínculo com os profissionais e isso fazia com que o tratamento fosse mais leve para ambos.

Houve ainda processos de estruturação compartilhada de diagnósticos e terapêuticas. O NASF AB-1 se orienta através dos preceitos da clínica ampliada, tendo sido criado para abranger uma compreensão maior entre o binômio saúde-doença, fornecendo assim, uma ampla visão do ser humano. Então, cada profissional contribui com sua ciência não de forma recortada e parcial, mas sim de forma conjunta, podendo se ater a todos os aspectos da saúde do usuário. Vivenciando um modo de fazer clínica de forma diferenciada e benéfica para os indivíduos que ali são assistidos. "Este compartilhamento vai tanto na direção da equipe de saúde, dos serviços de saúde e da ação intersetorial, como também do compartilhamento com os usuários" (BRASIL, 2008).

Logo, a maneira que os profissionais do NASF AB-1 trabalhavam era com reuniões com todos os profissionais envolvidos no cuidado, sendo realizadas a cada final do mês ou quando algum profissional sentia necessidade de uma intervenção que fosse pontual e necessária e que envolvesse todos. Essas reuniões serviam para compartilhar diagnósticos e terapêuticas no sentido de contribuir da melhor forma para que uma determinada demanda fosse solucionada, vale pontuar que essas reuniões se estendiam para a equipe ESF onde a demanda existia e para os usuários, com o objetivo de maior ganho integral.

Ressaltando que nem sempre é fácil se despir da autoimagem que cria-se em torno do profissional de saúde dentro do serviço, aquele que lida apenas no seu campo de trabalho sem querer intervir no campo do outro, por medo ou vaidade. O dispositivo NASF AB veio para desmitificar tal ideia, fazendo com que as trocas entre profissionais de forma ética fossem valorizada e utilizada em favor do usuário, e isso era que acontecia com os profissionais desse estágio, todos juntos com um só intuito de ajudar a solucionar de forma rápida e satisfatória o problema do paciente.

Nesse sentindo, as experiências vividas com os profissionais de outras áreas e com os usuários faz-se rica e importante para a formação acadêmica de quem pretende trabalhar na área. Portanto, tais vivências assumiram um lugar de reiteração e incentivo para a aquisição de conhecimentos e prática humanizada da profissão.

\section{CONCLUSÃO}

O Núcleo Ampliado de Saúde da Família e Atenção Básica opera, então, como sustentáculo às equipes de saúde da família na atenção primária fortalecendo e auxiliando nas

\section{E - book Pesquisa e Tecnologia: Protagonismo e Inovações}


políticas públicas de saúde no país. Seu trabalho é desenvolvido através de ações de prevenção de doenças e promoção de saúde para os usuários que são assistidos pelo equipamento, viabilizando ainda, matriciamento para as equipes conseguirem resolver as questões sem dependência de tais profissionais.

Contudo, cabe destacar que os NASF's AB mesmo sendo um dispositivo de ganho coletivo ainda enfrenta dificuldades em seu trabalho, como o modelo biomédico que respalda a formação dos profissionais de saúde ainda no binômio saúde-doença e muitas vezes se mostra como entrave para que os resultados sejam mais satisfatórios e rápidos, denunciando assim, uma cultura ainda biologicistas em que os futuros profissionais são impostos dentro da academia.

Então, a partir de ponderações empreendidas, entende-se que é necessário refletir sobre alguns aspectos do estágio, como: expandir a compreensão do papel de cada ator social nos espaços de intervenção; ampliar a interlocução no local de ensino e no cenário da prática; organizar o suporte para a mediação, entre Instituições de Ensino Superior (IES) e estágios para garantir uma prática profissional mais próxima da realidade; perceber as necessidades e expectativas das pessoas envolvidas no processo.

Com essa experiência, pode-se dizer que para poder realizar um trabalho eficiente na Atenção Básica, o primeiro passo é ter clareza a respeito do tipo de serviço a ser desenvolvido, não deve ser apenas clínico, ou seja, aquele tradicional. É preciso refletir e agir em favor de uma concepção de clínica ampliada. Ademais, é oportuno realizar projetos, desenvolvendo uma prática única e ao mesmo tempo plural, em proveito dos individuos e comunidades assistidas e referenciadas.

\section{REFERÊNCIAS}

BRASIL. Portaria $n^{\circ}$ 2.436, de 21 de setembro de 2017. Aprova a Política Nacional de Atenção Básica, estabelecendo a revisão de diretrizes para a organização da Atenção Básica, no âmbito do Sistema Único de Saúde (SUS). Brasília, DF: Ministério da Saúde, 2017. Acesso em: 13 maio, 2021.

CAMPOS, R. O. O encontro trabalhador-usuário na atenção à saúde: uma contribuição da narrativa psicanalítica ao tema do sujeito na saúde coletiva. Ciência \& Saúde Coletiva, v. 10, p. 573-583, 2005. Disponível em: Acesso em: 30 de março 2020.

CURY, R. S.; RODACOSKI, G. C.; SANTOS, C. L. Ampliação das atividades das equipes NASF-AB em saúde mental. Revista de Saúde Pública do Paraná, v. 2, p. 76-91, 2019.

\section{E - book Pesquisa e Tecnologia: Protagonismo e Inovações}


FERREIRA, J. S.; LADEIA, L. E. G. As Implicações da Política Nacional de Atenção Básica (PNAB) na Dinâmica dos Serviços de Saúde. ID on line REVISTA DE PSICOLOGIA , v. 12, n. 42, pág. 681-695, 2018. Disponível em: <https://idonline.emnuvens.com.br/id/article/view/1358>. Acesso em 10 jun. 2021.

FERRI, S. M. N.; ALMEIDA, M. C. P.; PEREIRA, M. J. B.; MISHIMA, S. M.; CACCIABAVA, M. C. G. As tecnologias leves como geradoras de satisfação em usuários de uma unidade de saúde da família. Interface Comunicação, Saúde, Educação, Botucatu, v. 11, n. 23, p. 515-529, 2007. Disponível em: <https://www.scielo.br/j/icse/a/J4NtsS774kLwwFKYV7JJs7g/abstract/?lang=pt>. Acesso em: 18 jun. 2021.

GIOVANELlA, L. Atenção básica ou atenção primária à saúde? Cadernos de Saúde Pública, v. 34, n. 8, 2018. DOI: 10.1590/0102-311X00029818. Acesso em: 22 jun. 2021.

RODRIGUES, L. B. B. et al. A atenção primária à saúde na coordenação das redes de atenção: uma revisão integrativa. Ciência \& Saúde Coletiva, v. 19, n 2, p. 343-352, 2014. Disponível em: https://www.scielo.br/j/csc/a/nBKRxhLTPkdp489zfNGhKnt/abstract/?lang=pt. Acesso em: 15 agosto 2021.

GIRÃO, V. M.; GOMES, E. M.; MAIA, A. H. N. Os Desafios para a Promoção da Saúde diante da nova Política Nacional da Atenção Básica: um relato de experiência. Encontro de Extensão, Docência e Iniciação Científica (EEDIC), v. 7, 2020. Disponível em: http://publicacoesacademicas.unicatolicaquixada.edu.br/index.php/eedic/article/view/4273.

Acesso em: 28 jun. 2021.

GONÇALVES, R. M. A. et al. Estudo do trabalho em núcleos de apoio à saúde da família (NASF), São Paulo, Brasil. Revista Brasileira de Saúde Ocupacional, v. 40, p. 59-74, 2015. Disponível em: <https://www.scielo.br/j/rbso/a/mNVPBPGdkVY9GKnrH78Q5nf/?lang=pt>. Acesso em: 15 de fev. 2019.

MENDONÇA, T. C. P. As oficinas na saúde mental: relato de uma experiência na internação. Psicologia: ciência e profissão, v. 25, p. 626-635, 2005.

SOUZA, F. L. D. et al. Implantação do Núcleo de Apoio à Saúde da Família: percepção do usuário. Saúde em Debate, v. 37, p. 233-240, 2013. Disponível em: <https://www.scielo.br/j/sdeb/a/pf5D6cHjYNWKZSypNNYMHnf/?lang=pt\&format=>. Acesso em 29 de jan. 2020.

OLIVEIRA, I. C.; ROCHA, R. M.; CUTOLO, L. R. A. Palavras sobre o NASF: relatando uma experiência acadêmica. Revista Brasileira de Educação Médica, v. 36, p. 574-580, 2012. Disponível em: <https://www.scielo.br/j/rbem/a/by4fkrjfjdtmhcXmBdLhzTc/?lang=pt\&format=html $>$. Acesso em:26 de jan de 2020.

VIANNA, H. M. Pesquisa em educação: a observação. Livro Editora Ltda. Brasília, DF. 2003. Inovações 